\title{
The relation between the factor systems of a group and those of an invariant subgroup
}

\author{
P M van den Broek \\ Institute for Theoretical Physics, University of Nymegen, the Netherlands
}

Received 18 December 1975, in final form 6 February 1976

\begin{abstract}
The relation between the factor systems of a group and the factor systems of an invariant subgroup is discussed both for PU and for PUA representations. The results are used to discuss the factor systems of a class of magnetic space groups.
\end{abstract}

\section{Introduction}

From the well-known results of Wigner and Bargmann it follows that the study of projective unitary (PU) and projective unitary-anti-unitary (PUA) representations of symmetry groups of physical systems is of great importance in physics. A natural consequence of dealing with PU and PUA representations is the study of their factor systems.

In this paper we will give a decomposition of the factor systems of a group which possesses an invariant subgroup. Such a decomposition is useful for the determination of a complete set of inequivalent factor systems, as we will show in $\$ \S 4$ and 5 . For factor systems of PU representations, decompositions have been given previously for two special cases: by Mackey (1958, see pp 303-4 especially) for the case where the group is a semi-direct product and by Backhouse and Bradley (1972) for the case where the invariant subgroup is of prime order. Mackey's result will turn out to be a special case of our result. Bradley and Wallis (1974) gave a decomposition for the factor systems of PUA representations for the case where the subgroup is of index two and is represented by unitary operators. Both the results of Backhouse and Bradley and those of Bradley and Wallis can easily be derived from our results.

The plan of the paper is as follows: in $\S 2$ we give some mathematical preliminaries to fix the notation, the main theorems are presented in $\$ 3$, and in $\$ \S 4$ and 5 we apply the results to those magnetic space groups which are semi-direct products of a black and white lattice and a point group with unitary elements.

\section{Preliminaries}

Let $G$ be a group and $G_{0}$ a subgroup of $G$ of index 1 or 2 . A PUA representation of $G$ with respect to $G_{0}$ is a mapping $D$ from $G$ into the operators on some Hilbert space $\mathscr{H}$ such that:

(i) the operator $D(g)$ is unitary if $g \in G_{0}$ and anti-unitary if $g \notin G_{0}$.

(ii) $D(g) D\left(g^{\prime}\right)=\sigma\left(g, g^{\prime}\right) D\left(g g^{\prime}\right)$ for all $g, g^{\prime} \in G$ for some mapping $\sigma: G \times G \rightarrow$ $U(1)$. 
(iii) $D(e)=I$, where $e$ is the identity of $G$ and $I$ is the identity operator on $\mathscr{H}$. The mapping $\sigma$ satisfies

$$
\sigma(g, e)=\sigma(e, g)=1 \quad \forall g \in G
$$

and

$$
\sigma\left(g, g^{\prime}\right) \sigma\left(g g^{\prime}, g^{\prime \prime}\right)=\sigma\left(g, g^{\prime} g^{\prime \prime}\right) \sigma^{g}\left(g^{\prime}, g^{\prime \prime}\right)
$$

where $\lambda^{8}$ is defined by

$$
\lambda^{g}= \begin{cases}\lambda & \text { if } g \in G_{0} \\ \lambda^{*} & \text { if } g \notin G_{0}\end{cases}
$$

and the asterisk denotes complex conjugation.

A mapping $\sigma: G \times G \rightarrow U(1)$ which satisfies equations (2.1) and (2.2) is called a factor system of $G$ with respect to $G_{0}$. In the following a factor system of $G$ shall always mean a factor system of $G$ with respect to $G_{0}$. If $D$ is a PUA representation of $G$ with factor system $\sigma$ and $c$ is a mapping from $G$ into $U(1)$ with $c(e)=1$ then $D^{\prime}(g)=c(g) D(g)$ is a PUA representation of $G$ with factor system

$$
\sigma^{\prime}\left(g_{1}, g_{2}\right)=\left[c\left(g_{1}\right) c^{g_{1}}\left(g_{2}\right) / c\left(g_{1} g_{2}\right)\right] \sigma\left(g_{1}, g_{2}\right) \text {. }
$$

Two factor systems $\sigma$ and $\sigma^{\prime}$ of $G$ are called equivalent if a mapping $c: G \rightarrow U(1)$ with $c(e)=1$ exists such that equation (2.4) holds. If $G_{0}$ is a subgroup of index 1 , i.e. $G=G_{0}$ then $D$ is a PU representation.

Now suppose $H$ is an invariant subgroup of $G$ and $K$ is the quotient group $G / H$. The elements of $K$ are the cosets of $G$ with respect to $H$. Elements of $H$ will be denoted by $a, b, c, \ldots$, and elements of $K$ by $\alpha, \beta, \gamma, \ldots$ Take for each coset $\alpha \in K$ a representative $r(\alpha)$, and let $r(\epsilon)=e$, if $\epsilon$ is the unit element of $K$. Then each element of $G$ can uniquely be written as $(a, \alpha)$ if we define

$$
(a, \alpha)=\operatorname{ar}(\alpha)
$$

Let $a^{\alpha}$ be defined by

$$
a^{\alpha}=r(\alpha) a r^{-1}(\alpha)
$$

and the mapping $m: K \times K \rightarrow H$ by

$$
r(\alpha) r(\beta)=m(\alpha, \beta) r(\alpha \beta) .
$$

From the associativity, it then follows

$$
m(\alpha, \beta) m(\alpha \beta, \gamma)=m(\alpha, \beta \gamma) m^{\alpha}(\beta, \gamma)
$$

and we also have

$$
\left(a^{\alpha}\right)^{\beta}=m(\beta, \alpha) a^{\beta \alpha} m^{-1}(\beta, \alpha) .
$$

The multiplication of the elements of $G$ is given by

$$
(a, \alpha)(b, \beta)=\left(a b^{\alpha} m(\alpha, \beta), \alpha \beta\right) .
$$

In the following we shall write $\lambda^{a}$ and $\lambda^{\alpha}$ for brevity if we mean $\lambda^{(a, \epsilon)}$ and $\lambda^{(\varepsilon, \alpha)}$ respectively. 
For the case where $G_{0}$ is a subgroup of index 2 we have to distinguish two possibilities:

(i) $H \cong G_{0}$. In this case $K$ has a subgroup $K_{0}$ of index 2 such that $(a, \alpha) \in G_{0}$ if and only if $\alpha \in K_{0}$.

(ii) $H \varsubsetneqq G_{0}$. Now $H$ has a subgroup $H_{0}$ of index 2 which equals $H \cap G_{0}$. Further, each coset of $G$ with respect to $H$ contains both elements of $G_{0}$ and elements of $G \backslash G_{0}$. Therefore we may choose the coset representatives $r(\alpha)$ to belong to $G_{0}$.

In the following we assume such choice has been made. Then $m(\alpha, \beta) \in H_{0}$ for all $a, \beta \in K$ and $(a, \alpha) \in G_{0}$ if and only if $a \in H$.

\section{The main theorems}

\section{Theorem 1.}

Let $\omega^{\prime}$ be a factor system of $G$. There exists an equivalent factor system $\omega$ of $G$ which decomposes as follows:

$$
\omega((a, \alpha),(b, \beta))=\gamma\left(a, b^{\alpha}\right) \gamma\left(a b^{\alpha}, m(\alpha, \beta)\right) \nu^{a b}(\alpha, \beta) P^{a}(\alpha, b)
$$

where $\gamma$ is a factor system of $H$ with respect to $H_{0}, \nu$ is a mapping from $K \times K$ into $U(1)$ and $P$ is a mapping from $K \times H$ into $U(1)$ with the following properties:

$$
\begin{aligned}
& \nu(\epsilon, \alpha)=\nu(\alpha, \epsilon)=1 \\
& P(\epsilon, a)=P(\alpha, e)=1 \quad \forall \alpha \in K \\
& P(\alpha, b c)=P(\alpha, b) P^{b}(\alpha, c) \frac{\gamma\left(b^{\alpha}, c^{\alpha}\right)}{\gamma^{\alpha}(b, c)} \\
& P(\alpha \beta, c)=P\left(\alpha, c^{\beta}\right) P^{\alpha}(\beta, c) \frac{\nu^{c}(\alpha, \beta)}{\nu(\alpha, \beta)} \frac{\gamma\left(m(\alpha, \beta) c^{\alpha \beta} m^{-1}(\alpha, \beta), m(\alpha, \beta)\right)}{\gamma\left(m(\alpha, \beta), c^{\alpha \beta}\right)} \\
& \frac{\nu(\alpha, \beta) \nu(\alpha \beta, \gamma)}{\nu(\alpha, \beta \gamma) \nu^{\alpha}(\beta, \gamma)}=\frac{\gamma\left(m^{\alpha}(\beta, \gamma), m(\alpha, \beta \gamma)\right)}{\gamma(m(\alpha, \beta), m(\alpha \beta, \gamma))} P(\alpha, m(\beta, \gamma))
\end{aligned}
$$

Proof.

Define the mapping $c: G \rightarrow U(1)$ by $c(a, \alpha)=\omega^{\prime}((a, \epsilon),(e, \alpha))$ and the factor system $\omega$ of $G$ by $\omega\left(g, g^{\prime}\right)=\left[c(g) c^{g}\left(g^{\prime}\right) / c\left(g g^{\prime}\right)\right] \omega^{\prime}\left(g, g^{\prime}\right)$. Then $\omega((a, \epsilon),(e, \alpha))=1$. Let $D$ be a PUA representation of $G$ with factor system $\omega$. Then $D((a, \epsilon)) D((e, \alpha))=D((a, \alpha))$. Now

$$
\begin{aligned}
& \omega((a, \alpha),(b, \beta)) D\left(\left(a b^{\alpha} m(\alpha, \beta), \alpha \beta\right)\right) \\
& \quad=D((a, \alpha)) D((b, \beta))=D((a, \epsilon)) D((e, \alpha)) D((b, \epsilon)) D((e, \beta)) .
\end{aligned}
$$

On the other hand we have

$$
\begin{aligned}
\omega\left((a, \epsilon),\left(b^{\alpha}, \epsilon\right)\right) \omega\left(\left(a b^{\alpha}, \epsilon\right),(m(\alpha, \beta), \epsilon)\right) \omega^{a b}((e, \alpha),(e, \beta)) D\left(\left(a b^{\alpha} m(\alpha, \beta), \alpha \beta\right)\right) \\
\quad=\omega\left((a, \epsilon),\left(b^{\alpha}, \epsilon\right)\right) \omega^{a b}((e, \alpha),(e, \beta)) D\left(\left(a b^{\alpha}, \epsilon\right)\right) D((m(\alpha, \beta), \epsilon)) D((e, \alpha \beta)) \\
=\omega\left((a, \epsilon),\left(b^{\alpha}, \epsilon\right)\right) D\left(\left(a b^{\alpha}, \epsilon\right)\right) \omega((e, \alpha),(e, \beta)) D((m(\alpha, \beta), \alpha \beta)) \\
\quad=D((a, \epsilon)) D\left(\left(b^{\alpha}, \epsilon\right)\right) D((e, \alpha)) D((e, \beta)) .
\end{aligned}
$$


If we multiply these two relations on the left with $D^{-1}((a, \epsilon))$ and on the right with $D^{-1}((e, \beta))$ and combine them we obtain

$$
\begin{aligned}
D((e, \alpha)) D((b, \epsilon)) & \\
= & \frac{\omega^{a}((a, \alpha),(b, \beta))}{\omega^{a}\left((a, \epsilon),\left(b^{\alpha}, \epsilon\right)\right) \omega^{a}\left(\left(a b^{\alpha}, \epsilon\right),(m(\alpha, \beta), \epsilon)\right) \omega^{b}((e, \alpha),(e, \beta))} \\
& D\left(\left(b^{\alpha}, \epsilon\right)\right) D((e, \alpha)) .
\end{aligned}
$$

Since the numerical factor on the right-hand side depends on $\alpha$ and $b$ only we may denote it by $P(\alpha, b)$.

Let the mapping $\nu: K \times K \rightarrow U(1)$ be defined by $\nu(\alpha, \beta)=\omega((e, \alpha),(e, \beta))$ and let $\gamma$ be the restriction of $\omega$ to $H \times H$. Then $\gamma$ is a factor system of $H$, and equation (3.1) has been proved. Equation (3.2) follows from the definition of $\nu$ and equation (3.3) follows from equation (3.1) if we take $\alpha=\epsilon ; a=e$ and $b=e ; \beta=\epsilon$ respectively. Now from the definition of a factor system we have

$$
\begin{aligned}
\omega((a, \alpha)(b, \beta)) \omega\left(\left(a b^{\alpha} m(\alpha, \beta), \alpha \beta\right),(c, \gamma)\right) \\
=\omega\left((a, \alpha),\left(b c^{\beta} m(\beta, \gamma), \beta \gamma\right)\right) \omega^{(a, \alpha)}((b, \beta),(c, \gamma)) .
\end{aligned}
$$

If we take $\beta=\epsilon$ in this equation and use equation (3.1), the result is equation (3.4). In the same way equation (3.5) follows if we take $a=b=e$ and $\gamma=\epsilon$. Finally equation (3.6) follows if we take $a=b=c=e$. This proves the theorem.

The opposite of the theorem above is also true:

\section{Theorem 2.}

Let $\gamma$ be a factor system of $H$ with respect to $H_{0}$. Let $\nu$ and $P$ be mappings from $K \times K$ and $K \times H$ respectively into $U(1)$ such that the equations (3.2), (3.3), (3.4), (3.5) and (3.6) are satisfied. Then the mapping $\omega: G \times G \rightarrow U(1)$ defined by equation (3.1) is a factor system of $G$.

The proof of this theorem is a straightforward calculation and is therefore omitted.

The question when two factor systems decomposed as in equation (3.1) are equivalent is answered by the following theorem.

\section{Theorem 3.}

A factor system $\omega$ decomposed as in equation (3.1) is equivalent with the trivial factor system if and only if there exist mappings $d: H \rightarrow U(1)$ and $e: K \rightarrow U(1)$ with $d(e)=e(\epsilon)=1$ such that $\gamma, P$ and $\nu$ can be written as

$$
\begin{aligned}
& \nu(\alpha, \beta)=\frac{e(\alpha) e^{\alpha}(\beta)}{d(m(\alpha, \beta)) e(\alpha \beta)} \\
& P(\alpha, b)=\frac{e(\alpha) d^{\alpha}(b)}{d\left(b^{\alpha}\right) e^{b}(\alpha)}
\end{aligned}
$$

and

$$
\gamma(a, b)=\frac{d(a) d^{a}(b)}{d(a b)}
$$


Proof.

If $\omega$ is equivalent with the trivial factor system then there exists a mapping $c: G \rightarrow U(1)$, with $c((e, \epsilon))=1$ such that

$\gamma\left(a, b^{\alpha}\right) \gamma\left(a b^{\alpha}, m(\alpha, \beta)\right) \nu^{a b}(\alpha, \beta) P^{a}(\alpha, b)=\frac{c((a, \alpha)) c^{(\alpha, \alpha)}((b, \beta))}{c\left(\left(a b^{\alpha} m(\alpha, \beta), \alpha \beta\right)\right)}$.

If we define $d$ and $e$ by $d(a)=c((a, \epsilon))$ and $e(\alpha)=c((e, \alpha))$ then it follows from equation (3.10) with $b=e$ and $\alpha=\epsilon$

$$
c((a, \beta))=d(a) e^{a}(\beta) .
$$

Now the equations (3.7), (3.8) and (3.9) follow immediately from (3.10) and (3.11) by taking the appropriate elements equal to the unit element.

On the other hand, if equations (3.7), (3.8) and (3.9) hold for some mappings $d: H \rightarrow U(1)$ and $e: K \rightarrow U(1)$ with $d(e)=e(\epsilon)=1$ and the mapping $c: G \rightarrow U(1)$ is defined by equation (3.11) then it is easily verified that equation (3.10) holds, which means that $\omega$ is equivalent with the trivial factor system.

\section{The factor systems of black and white lattices}

In this section we shall apply the decomposition given in the preceding section to derive the factor systems of the three-dimensional black and white lattices. Let $T_{0}$ be a three-dimensional lattice and let $a_{1}, a_{2}$ and $a_{3}$ be basis translations for $T_{0}$. Suppose $T$ is ablack and white lattice for which $T_{0}$ is the ordinary sublattice. $T$ is then determined by $T_{0}$ and an extra translation $a$ with the property $2 a \in T_{0}$.

The inequivalent factor systems of $T_{0}$ are obtained by Backhouse (1970) and are given by

$$
\gamma\left(t, t^{\prime}\right)=\exp \left(-2 \pi i t^{\mathrm{T}} A t^{\prime}\right)
$$

where $t$ stands for the translation $t=t_{1} a_{1}+t_{2} a_{2}+t_{3} a_{3}$ and for the column vector with entries $t_{1}, t_{2}$ and $t_{3}$ at the same time, $t^{\mathrm{T}}$ is the transpose of $t$ and $A$ is a matrix of the form

$$
A=\left(\begin{array}{lll}
0 & 0 & 0 \\
p & 0 & 0 \\
r & s & 0
\end{array}\right)
$$

where $p, r, s \in[0,1)$.

The quotient group $T / T_{0}$ equals $C_{2}$, consisting of the elements $\epsilon$ and $\alpha$, with $\alpha^{2}=\epsilon$. We choose $r(\alpha)=a$, so $m(\alpha, \alpha)=2 a$. For each factor system $\gamma$ of $T_{0}$ we have to solve $P(\alpha, t)$ and $\nu(\alpha, \alpha)$ from equations (3.4), (3.5) and (3.6) which now take the form

$$
\begin{array}{ll}
P\left(\alpha, t+t^{\prime}\right)=P(\alpha, t) P\left(\alpha, t^{\prime}\right) \frac{\gamma\left(\boldsymbol{t}, \boldsymbol{t}^{\prime}\right)}{\gamma^{*}\left(\boldsymbol{t}, \boldsymbol{t}^{\prime}\right)} & \forall t, t^{\prime} \in T_{0} \\
\gamma(\boldsymbol{t}, 2 a)=\gamma(2 a, t) \quad \forall t \in T_{0} & \\
\frac{\nu(\alpha, \alpha)}{\nu^{*}(\alpha, \alpha)}=P(\alpha, 2 a) &
\end{array}
$$

From equation (4.3) it follows that $\gamma^{2}$ must be equivalent to the trivial factor system of $T_{0}$. This restricts the possible values of $p, r$ and $s$ to 0 and $\frac{1}{2}$. From these eight 
possibilities only two satisfy equation (4.4). One of them is of course the trivial factor system $(p=r=s=0)$ and the other is given in table 1 .

Table 1.

\begin{tabular}{cccc}
\hline $2 a$ & $p$ & $r$ & $s$ \\
\hline$a_{1}$ & 0 & 0 & $\frac{1}{2}$ \\
$a_{2}$ & 0 & $\frac{1}{2}$ & 0 \\
$a_{3}$ & $\frac{1}{2}$ & 0 & 0 \\
$a_{1}+a_{2}$ & 0 & $\frac{1}{2}$ & $\frac{1}{2}$ \\
$a_{1}+a_{3}$ & $\frac{1}{2}$ & 0 & $\frac{1}{2}$ \\
$a_{2}+a_{3}$ & $\frac{1}{2}$ & $\frac{1}{2}$ & 0 \\
$a_{1}+a_{2}+a_{3}$ & $\frac{1}{2}$ & $\frac{1}{2}$ & $\frac{1}{2}$ \\
\hline
\end{tabular}

For the two remaining factor systems $\gamma$ of $T_{0}$ we have $\gamma\left(t, t^{\prime}\right) / \gamma^{*}\left(t, t^{\prime}\right)=1$, so the solutions of equation (4.3) are just the one-dimensional unitary representations of $T_{0}$ which are well known. For each solution $P(\alpha, t)$ of equation (4.3) there are two solutions $\nu(\alpha, \alpha)$ of equation (4.5). The inequivalent factor systems of $T$ can now be derived with theorem 3 in a straightforward manner. It turns out that there are only two inequivalent factor systems of $T$. A nontrivial one is given by the nontrivial $\gamma$ and $P(\alpha, t)=\nu(\alpha, \alpha)=1$.

\section{Factor systems of a class of magnetic space groups}

As a second application let us consider a magnetic space group $G$ which is the semi-direct product of a point group $P$ and the invariant subgroup $T$ of translations, which forms a black and white lattice, and let the nonmagnetic subgroup of $G$ also be a semi-direct product. The point group $P$ acts in the natural way on $T$ and on the ordinary sublattice $T_{0}$ and leaves both $T$ and $T_{0}$ invariant. Of the 517 magnetic space groups which are based on black and white lattices, 110 are of this type.

The problem now is to solve the equations (3.2)-(3.6) where $H$ is the black and white lattice $T$ and $K$ is the point group $P$. The elements of $P$ may now be chosen to be the coset representatives of $G$ with respect to $H$. Equation (3.6) now tells us that $\nu$ is a factor system of $P$, and according to theorem 3 we can take this factor system to be real, since every factor system of a point group is equivalent to a real factor system. Therefore $\nu$ cancels from equation (3.5) and we only have to solve $P(R, t)$ from the equations

$$
\dot{P}\left(R, t+t^{\prime}\right)=P\left(R, t_{1}\right) P^{t_{1}}\left(R, t_{2}\right) \frac{\gamma\left(R t_{1}, R t_{2}\right)}{\gamma\left(t_{1}, t_{2}\right)} \quad \forall t_{1}, t_{2} \in T, \forall R \in P
$$

and

$$
P\left(R_{1} R_{2}, t\right)=P\left(R_{1}, R_{2} t\right) P\left(R_{2}, t\right) \quad \forall t \in T, \forall R_{1}, R_{2} \in P .
$$

The general solution $P_{0}(R, t)$ of equations (5.1) and (5.2) can be written as

$$
P_{0}(R, t)=P_{1}(R, t) P(R, t)
$$


where $P_{1}(R, t)$ is a particular solution of equations (5.1) and (5.2) and $P(R, t)$ is the general solution of (5.2) and the equation

$$
P\left(R, t_{1}+t_{2}\right)=P\left(R, t_{1}\right) P^{t_{1}}\left(R, t_{2}\right) \quad \forall t_{1}, t_{2} \in T, \forall R \in P .
$$

The solutions of equation (5.4) are just the one-dimensional UA representations of $T$.

The reciprocal lattice $T_{0}^{*}$ of $T_{0}$ is spanned by $b_{1}, b_{2}$ and $b_{3}$ where

$$
b_{i}=\frac{2 \pi a_{j} \otimes a_{k}}{a_{i} \cdot\left(a_{j} \otimes a_{k}\right)}
$$

where $j=(i+1) \bmod (3)$ and $k=(i+2) \bmod (3)$. As is well known the onedimensional unitary representations of $T_{3}$ are given by $\Delta_{k}(t)=\mathrm{e}^{\mathrm{ik} \cdot t}$ where $k$ ranges through the first Brillouin zone of the reciprocal lattice:

$$
\boldsymbol{k}=\alpha_{1} \boldsymbol{b}_{1}+\alpha_{2} \boldsymbol{b}_{2}+\alpha_{3} \boldsymbol{b}_{3} \quad \text { where } \alpha_{i} \in[0,1) .
$$

After a short calculation we find that the one-dimensional UA representations of $T$ are labelled by a pair $(\boldsymbol{k}, z)$ where $z \in U(1)$ and $\boldsymbol{k} \in \Lambda(a) . \Lambda(a)$ denotes the set consisting of those four vectors $\boldsymbol{k}$ from the first Brillouin zone that have the properties that $2 \boldsymbol{k}$ is a reciprocal lattice vector and $\mathrm{e}^{\mathrm{ik} \cdot 2 a}=1$. For instance if $2 a=a_{1}$ then

$$
\Lambda(\boldsymbol{a})=\left\{0, \frac{\boldsymbol{b}_{2}}{2}, \frac{\boldsymbol{b}_{3}}{2}, \frac{\boldsymbol{b}_{2}}{2}+\frac{\boldsymbol{b}_{3}}{2}\right\}
$$

The one-dimensional UA representations of $T$ are then given by

$$
D_{k, z}(t)=\mathrm{e}^{\mathrm{i} k . t} \quad D_{k, z}(t+a)=z \mathrm{e}^{\mathrm{i} k . t} \quad \forall t \in T_{0} .
$$

The general solution of equation (5.4) is given by $P(R, t)=D_{k(R), z(R)}(t)$. The equations which $k(R)$ and $z(R)$ must satisfy if $P(R, t)$ satisfies equation $(5.2)$ are

$$
k\left(R_{1} R_{2}\right)=R_{2}^{-1} k\left(R_{1}\right)+k\left(R_{2}\right)+K\left(R_{1}, R_{2}\right) \quad \forall R_{1}, R_{2} \in P
$$

and

$$
z\left(R_{1} R_{2}\right)=z\left(R_{1}\right) z\left(R_{2}\right) \mathrm{e}^{\mathrm{i} \boldsymbol{a}\left[\mathrm{k}\left(k\left(R_{1} R_{2}\right)-k\left(R_{1}\right)-k\left(R_{2}\right)-K\left(R_{1}, R_{2}\right)\right]\right.} \quad \forall R_{1}, R_{2} \in P .
$$

Here $\boldsymbol{K}$ is a reciprocal lattice vector which depends on $R_{1}$ and $R_{2}$. Equation (5.7) is analogous to the equation derived by Backhouse (1970) for the nonmagnetic symmorphic space groups. In that case however $\boldsymbol{k}$ ranges through the whole first Brillouin zone, while here $\boldsymbol{k}$ is restricted to the four vectors in $\Lambda(\boldsymbol{a})$, which makes equation (5.7) rather easy to solve. Only those solutions of equation (5.7) for which $\sigma\left(R_{1}, R_{2}\right)=$ $\exp \left(\mathrm{i} a \cdot \boldsymbol{K}\left(R_{1}, R_{2}\right)\right)$ is a trivial factor system of $P$ have to be considered. For those solutions $z(R)$ must form a projective representation of $P$ with factor system $\sigma^{\prime}\left(R_{1}, R_{2}\right)=\exp \left\{\mathrm{i} a .\left[K\left(R_{1}, R_{2}\right)-k\left(R_{1}, R_{2}\right)+k\left(R_{1}\right)+k\left(R_{2}\right)\right]\right\}$. This factor system is equivalent with $\sigma$. Note that $\boldsymbol{k}(R), \boldsymbol{a}=0$ if $2 \boldsymbol{a}$ is equal to $\boldsymbol{a}_{1}, \boldsymbol{a}_{2}$ or $\boldsymbol{a}_{3}$.

Let us now turn our attention to the problem of finding a solution to equations (5.1) and (5.2). If $\gamma$ is the trivial factor system of $T$ then of course $P_{1}(R, t)=1$ solves equations (5.1) and (5.2). Therefore consider the case where $\gamma$ is the nontrivial factor system of $T$.

The factor system $\gamma^{R}$ of $T$ defined by $\gamma^{R}\left(t, t^{\prime}\right)=\gamma\left(R t, R t^{\prime}\right) \gamma^{-1}\left(t, t^{\prime}\right)$ is equivalent with the trivial factor system for each $R \in P$, since $T$ has only two inequivalent factor systems. Therefore we can find a mapping $c_{R}: T \rightarrow U(1)$ with $c_{R}\left(t_{0}\right)=1\left(t_{0}\right.$ is the unit 
element of $T$ ) and

$$
\gamma^{R}\left(t, t^{\prime}\right)=c_{R}(t) c_{R}^{t}\left(t^{\prime}\right) / c_{R}\left(t+t^{\prime}\right)
$$

$c_{R}$ is determined up to multiplication by a one-dimensional $U A$ representation of $T$. Moreover $c_{R_{1}}\left(R_{2} t\right) c_{R_{2}}(t) c_{R_{1} R_{2}}^{-1}(t)$ is a UA representation of $T$. Now it is easy to see that equations (5.1) and (5.2) have solutions if and only if the $c_{R}$ can be chosen such that $c_{R_{1}}\left(R_{2} t\right) c_{R_{2}}(t) c_{R_{1} R_{2}}^{-1}(t)=1$ for all $t \in T$ and for all $R_{1}, R_{2} \in P$. A solution is then given by $P_{1}(R, t)=c_{R}^{-1}(t)$.

Finally we have to apply theorem 3 in order to obtain the inequivalent factor systems. The factor systems obtained so far are given by $(\gamma, \nu, P)$ where $\gamma$ is one of the two inequivalent factor systems of $T, \nu$ belongs to a complete set of inequivalent real factor systems of $P$ and $P$ is a solution of equations (5.1) and (5.2). Let $\omega$ and $\omega^{\prime}$ be the factor systems of $G$ given by $(\gamma, \nu, P)$ and $\left(\gamma^{\prime}, \nu^{\prime}, P^{\prime}\right)$ respectively. $\omega$ and $\omega^{\prime}$ can be equivalent only if $\gamma=\gamma^{\prime}$ and $\nu=\nu^{\prime}$. If this is the case then

$$
P(R, t) P^{\prime-1}(R, t)=D_{k_{0}(R), z_{0}(R)}(t)
$$

for some $\boldsymbol{k}_{0} \in \Lambda(a)$ and $z_{0} \in U(1)$. $\omega$ and $\omega^{\prime}$ are now equivalent if and only if there exists a $\boldsymbol{k}_{1}$ in $\Lambda(a)$ and a one-dimensional unitary representation $d$ of $P$ such that

$$
\boldsymbol{k}_{0}(R)=\boldsymbol{k}_{1}-R^{-1} \boldsymbol{k}_{1}+\boldsymbol{K} \quad \forall R \in P
$$

and

$$
z_{0}(R)=d^{2}(R) \mathrm{e}^{\mathrm{i} R k_{0} \cdot \boldsymbol{a}} \quad \forall R \in P .
$$

This shows how to obtain a complete set of inequivalent factor systems of $G$.

\section{Acknowledgment}

I would like to thank Dr T Janssen for reading the manuscript.

\section{References}

Backhouse N B 1970 Q. J. Math. 21 277-95

Backhouse N B and Bradley C J 1972 Q. J. Math. 23 225-38

Bradley C J and Wallis D E 1974 Q. J. Math. 25 85-99

Mackey G W 1958 Acta Math. 99 265-311 